\title{
Distinguishing lard from other animal fats in admixtures of some vegetable oils using liquid chromatographic data coupled with multivariate data analysis
}

\begin{abstract}
Detection of animal fat adulterants in vegetable oils is of great importance from commercial and health perspectives. Distinguishable identification of lard contamination in some vegetable oils has been attempted in this study. Vegetable oils, namely palm oil (PO), palm kernel oil (PKO), and canola oil (CLO), were spiked with different proportions of animal fats, such as lard (GLD), beef tallow (BT), and chicken fat (CF). High-performance liquid chromatographic (HPLC) analyses were performed to monitor the triacylglycerol (TAG) compositional changes in the oil samples before and after adulteration. The results showed that qualitative determination of lard contamination in PKO was possible by a visual comparison of TAG profiles of PKO adulterated with different animal fats with those of the animal fats. This approach was not useful for PO and CLO. However, by subjecting liquid chromatographic data to multivariate procedures, distinguishable grouping of lardcontaminated samples was achieved for all three oils.
\end{abstract}

Keyword: Adulteration, Animal fat, High-performance liquid chromatography, Lard, Vegetable oils, Multivariate data analysis 